Annuaire suisse de politique de développement

13 | 1994

Annuaire Suisse - Tiers Monde 1994

\title{
Santé ou développement?. Pour une stratégie de coopération mieux orientée
}

Jean-Pierre Gontard, Antoine Degrémont, Eric Burnier, Nicolaus Lorenz et Marcel Tanner

\section{OpenEdition}

1 Journals

Édition électronique

URL : http://journals.openedition.org/aspd/1062

DOI : $10.4000 /$ aspd. 1062

ISSN : 1663-9669

Éditeur

Institut de hautes études internationales et du développement

Édition imprimée

Date de publication : 1 janvier 1994

Pagination : 232-236

ISSN : 1660-5934

\section{Référence électronique}

Jean-Pierre Gontard, Antoine Degrémont, Eric Burnier, Nicolaus Lorenz et Marcel Tanner, «Santé ou développement? ? Pour une stratégie de coopération mieux orientée ", Annuaire suisse de politique de développement [En ligne], 13 | 1994, mis en ligne le 19 décembre 2012, consulté le 08 septembre 2020. URL : http://journals.openedition.org/aspd/1062 ; DOI : https://doi.org/10.4000/aspd.1062 


\section{Santé ou développement? Pour une stratégie de coopération mieux orientée}

\section{D'Alma Ata à Copenhague - 1978-1995}

L'économique (la production, la consommation) a été pendant longtemps au centre des préoccupations des agences de développement. Essayons de modifier ce schéma et remettons l'homme au milieu du village, l'individu dans son contexte social. Que la santé, au sens large de bien-être physique, mental et social (définition de l'OMS) devienne une fin en soi et ne soit plus un simple moyen au service du développement économique.

A Alma Ata (1978), la communauté internationale a défini les huit composantes essentielles de tout programme de santé de base. Elle a aussi reconnu l'importance de la participation communautaire pour la définition et la mise en oeuvre des programmes de développement sanitaire. Mais elle est restée timide en affirmant seulement:"La promotion et la protection de la santé des peuples sont la condition sine qua non d'un progrès économique et social soutenu...".

Espérons qu'à Copenhague, en 1995, lors du Sommet mondial sur le développement social organisé par les Nations Unies, la déclaration adoptée ressemblera à ceci:"Tout Progrès économique et social vise à assurer à l'être humain, d'où qu'il soit, le niveau le plus élevé possible de bien-être physique, mental et social".

Comment traduire ce renversement de priorités en termes stratégiques pour orienter la coopération suisse dans le domaine sanitaire? Essayons de préciser quelles seraient les conséquences pratiques d'un tel changement de priorité. Elles seraient nombreuses, à la fois sur le secteur sanitaire proprement dit et sur les autres secteurs qui lui sont directement liés.

Si nous remettons l'homme au centre dans son contexte social, nous sommes amenés immanquablement à tenir compte des besoins et du potentiel d'une plus large gamme de partenaires; en cercles concentriques autour de lui, nous trouvons: les ménages et les familles; la communauté et tous ses sous-groupes; 
les villages et les quartiers; les collectivités administratives locales; le district et ses services techniques ("l'unité fonctionnelle"); la région et l'Etat central.

L'homme dans son contexte social c'est aussi la prise en compte d'entités comme les minorités ethniques et culturelles, les migrants.

\section{Des ménages à l'état central}

Du côté suisse, les interventions dans le domaine de la santé se font par l'intermédiaire des ONG, du bilatéral public avec les régisseurs, du multilatéral et de quelques ONG internationales spécialisées. Chacun de ces types d'organismes correspond à un niveau de partenaire particulier. Les interventions publiques suisses devraient se répartir selon le schéma suivant:

A - Niveau primaire

Ménages

Communautés

Villages

Quartiers des villes

< prestations techniques > avec

ONG suisses

ONG locales

Migrants, minorités

B - Niveau secondaire

Districts ruraux $<$ prestations techniques > $\begin{aligned} & \text { Bilatéral } \\ & \text { avec } \\ & \text { régisseurs }\end{aligned}$

Districts urbains
Villes moyennes

C - Niveau tertiaire

Capitales

Hôpitaux centraux Ministères

< prestations techniques > Multilatéral

D-Niveau étatique

Normes du gouvernement

Systèmes nationaux

Facultés

Recherche

Innovation

$<\begin{gathered}\text { prestations à fortes } \\ \text { composantes } \\ \text { intellectuelles } \\ \text { et politiques }\end{gathered}>$

Hautes Ecoles suisses

Milieux privés ONG internationales spécialisées 
Les ressources publiques suisses devraient être réparties entre les divers niveaux en tenant compte non seulement des besoins objectifs des partenaires en matière de coopération sanitaire, mais aussi de la capacité suisse à les satisfaire.

Les programmes impliquant une approche pluridisciplinaire et favorisant la coopération intersectorielle seraient favorisés.

\section{A - Niveau primaire}

Les besoins du niveau A sont infinis. La Confédération devrait cofinancer les programmes des ONG suisses dans la limite de leur capacité à mobiliser d'autres ressources et de leur volonté de respecter les lignes directrices de la DDA dans le domaine sanitaire. La DDA ne devrait par exemple pas cofinancer d'ONG suisses travaillant de façon autonome sans partenaire sur le terrain. Elle ne devrait pas non plus cofinancer d'ONG qui ne seraient actives que dans le domaine curatif à l'exclusion du préventif. Le renforcement des institutions locales de santé communautaire et leur implication dans le processus de décision seraient essentiels comme critères de collaboration.

\section{B - Niveau secondaire}

Le niveau district, au sens de la déclaration de Harare (1988), devrait être le niveau privilégié de collaboration entre la Suisse et ses partenaires et devrait recevoir une plus grande part des ressources de la DDA. Pour l'OMS, un district sanitaire, rural ou urbain, comprend entre $50^{\prime} 000$ et 500 '000 habitants. La coopération bilatérale se prête bien à un travail intersectoriel, préventif et curatif, réaliste et innovateur, avec une grande variété de partenaires dans un cadre géographique et administratif comme celui du district, ou d'une grappe de districts.

Les programmes de développement sanitaire à ce niveau, tels que nous les concevons, sont des programmes de longue durée, entrecoupés d'évaluations épidémiologiques, institutionnelles et financières. Ils comprennent un paquet minimum de techniques (médicales et non médicales) qui, une fois défini localement, n'est plus négociable. Ce paquet reprend en gros les huit composantes des politiques de SSP, telles qu'elles ont été définies à Alma Ata: éducation sanitaire, nutrition, eau, assainissement, protection maternelle et infantile $-y$ compris planification familiale -, vaccination et contrôle des grandes épidémies, traitement des maladies et lésions courantes, médicaments essentiels.

Chacune de ces activités techniques implique des moyens humains et financiers spécifiques. Il faudrait éviter de confier des responsabilités trop élevées à des groupes aux capacités trop limitées.

\section{C - Niveau tertiaire}

Dans la mesure où la Suisse peut influencer les institutions multilatérales qu'elle contribue à financer (Banque mondiale, banques régionales, PNUD, institutions spécialisées), elle devrait favoriser le financement par ces institutions de program- 
mes visant la réhabilitation du niveau technique tertiaire, comme les hôpitaux régionaux (plutôt que l'exécution de nouvelles constructions). En effet, si les niveaux primaires et secondaires fonctionnent bien, la quantité de personnes référées à juste titre vers les installations régionales ou nationales va augmenter dans la mesure où les prestations offertes par ces installations sont d'une qualité technique adéquate. Par ailleurs, la Suisse devrait encourager les organisations multilatérales à participer activement aux programmes nationaux de réforme des Ministères et autres institutions publiques et privées jouant un rôle dans la gestion de la santé publique.

\section{D - Niveau étatique}

L'inversion des priorités, soit l'économie au service de la santé et non la santé au service de l'économie, implique des changements dans le rôle des institutions nationales au Nord comme au Sud. La Suisse doit soutenir financièrement et techniquement les Etats qui entreprennent des réformes de leurs systèmes afin de mieux accomplir ces nouvelles tâches.

L'efficacité de la prévention et la qualité des soins administrés par les secteurs privé, public ou non-lucratif. doivent être garantis par l'Etat, de même la qualité de la formation initiale et permanente des personnels privés ou publics. II revient à l'Etat d'établir et de faire respecter de nouvelles normes en matière d'environnement et d'éthique; l'Etat doit réformer les politiques pharmaceutiques qui sont le plus souvent très faibles et contre-productives. Les innovations spontanées de type mutuelles ou de type assurances, doivent être encouragées, mais dans un cadre harmonieux respectant les principes d'équité. La recherche épidémiologique et l'utilisation de ses résultats par des partenaires peu habitués à le faire est aussi une priorité, surtout en période de crise financière.

La Suisse doit encourager ses hautes-écoles à travailler dans ces domaines en collaboration avec des partenaires de même nature dans le Sud ou sous contrat avec les Etats du Sud. Ces recherches appliquées devraient impliquer les personnels des niveaux primaire et secondaire. Certaines institutions internationales spécialisées non gouvernementales pourraient aussi utiliser des fonds suisses, pour de la recherche appliquée et non pour des opérations, car il ne s'agit pas de financer des programmes trop verticaux. Le secteur privé suisse peut également jouer un rốle dans le transfert de connaissances et de capacité de recherche et de développement (par exemple: Institut suisse des sérums, ...). "La stratégie suisse pour l'encouragement de la recherche d'autres pays en développement" DDA Académie suisse des sciences naturelles, juillet 1993, pourrait servir de cadre à bon nombre de ces activités.

Dans les cas particuliers de situations d'instabilité politique et de troubles internes, de plus en plus fréquents, il est important d'utiliser toute la gamme des institutions suisses pour maintenir l'appui aux populations aux niveaux primaire et secondaire (ONG, coopération bilatérale, aide en cas de catastrophe, CICR).

Dans le cas particulier des pays qui reçoivent beaucoup d'aide extérieure, la Suisse est particulièrement bien placée pour prendre des initiatives visant à 
renforcer la coordination entre la politique nationale de santé et les stratégies des divers bailleurs de fonds.

Parmi les grands programmes verticaux internationaux, la DDA devrait donner priorité à ceux dont les objectifs couvrent des domaines de risque (par exemple santé urbaine) ou des groupes à risque (enfants de moins de cinq ans), plutôt que des maladies ou des méthodes (malaria, schistosomiase ou même sida).

\section{Utilisation optimale des compétences}

La répartition des crédits publics entre les niveaux décrits devrait se faire après consultation interdisciplinaire, et non à l'intérieur d'un groupe qui soit trop exclusivement médical ou trop exclusivement sur la coopération. Un nouveau "groupe santé" indépendant, constitué par une gamme très ouverte de professionnels suisses expérimentés, pourrait reprendre certaines des fonctions de l'ancien groupe santé, qui a joué un rôle très positif pour la mise au point de lignes directrices, l'aide à la décision, la capitalisation d'expériences, en particulier après les évaluations.

Un tel groupe permettrait de mieux faire le lien entre les milieux de la santé et les milieux de la coopération et susciterait un intérêt pour la politique suisse de coopération dans des milieux socio-professionnels importants.

Jean-Pierre Gontard, Antoine Degrémont, Eric Burnier, Nicolaus Lorenz, Marcel Tanner 